\title{
Processo de Implementação da Residência Multiprofissional em Saúde em um Hospital Universitário: Principais Avanços e Desafios
}

\author{
The Implementation of a Multidisciplinary Health Residency Program in a \\ University Hospital: Main Advances and Challenges
}

\author{
CARLA MOUSINHO FERREIRA LUCENA ${ }^{1}$ \\ ANA PAULA ROCHA DE SALES MIRANDA ${ }^{2}$ \\ PABLO LEONID CARNEIRO LUCENA ${ }^{3}$ \\ PATRÍCIABARRETO CAVALCANTI ${ }^{4}$ \\ SAIONARA FERREIRAARAÚJO DOS SANTOS ${ }^{5}$
}

RESUMO

Objetivo: Investigar o desenvolvimento do processo de implementação da Residência Multiprofissional em um hospital universitário. Material e Métodos: Pesquisa de natureza exploratória com caráter qualiquantitativo. Foram utilizados como instrumentos de coleta de dados: levantamentos documentais e bibliográficos e a aplicação de questionário junto aos preceptores e tutores do Programa. Os dados foram analisados a partir da literatura empreendida para consecução da pesquisa. Resultados: $89 \%(18)$ da amostra foi constituída por preceptores e $11 \%(3)$ tutores. Os participantes mencionaram que o processo de implementação foi permeado por dificuldades, dentre elas: a estrutura organizacional do Programa; o número de profissionais responsáveis por garantir o acompanhamento dos residentes; a ausência de normas e rotinas claras e eficientes; a crise enfrentada pelos HUs, a qual pôde ser constatada pelo alto índice de profissionais terceirizados, pela escassez de recursos humanos e materiais, além dos problemas infraestruturais; o envelhecimento dos profissionais da instituição; e a inexistência de integração entre a Residência Multiprofissional e a Residência Médica. Todavia, segundo os mesmos, foi possível avançar incitando a construção de uma nova concepção de trabalho em saúde, principalmente devido à contribuição do Projeto Terapêutico Singular, da realização de atividades de cunho educativo e da realização de práticas com valorização da intersetorialidade e integralidade. Conclusão: Considera-se que a experiência analisada projetou avanços importantes, pois engendrou ações de educação em saúde, intersetorialidade e reorganização de ações nas práticas profissionais. Contudo, ainda não conseguiu modificar os processos de trabalho, cuja ruptura exige um projeto articulado entre as distintas profissões e cooperação da gestão hospitalar.

DESCRITORES

Recursos Humanos.Capacitação Profissional.Educação Continuada.

\begin{abstract}
Objective: To investigate the implementation process of a multidisciplinary residency program in a university hospital (UH). Material and Methods: This was an exploratory study with qualitative and quantitative approaches. The data were collected using documentary and bibliographical surveys and a questionnaire submitted to program preceptors and tutors. The data were analyzed based on the literature surveyed to develop this study. Results: $89 \%(18)$ of the sample consisted of preceptors and $11 \%(3)$ tutors. The participants mentioned that the implementation process was permeated by difficulties, among them: an organizational structure of the Program; the number of professionals for viability or monitoring of residents; an absence of clear and efficient standards and routines; the crisis faced by UHs, which was evidenced by the high number of outsourced professionals; scarcity of human and material resources, and infrastructural problems; the aging of the institution's professionals; and the lack of integration between the Multidisciplinary Residency Program and the Medical Residency Program. However, according to them, it was possible to advance by encouraging the construction of a new conception of health work, mainly due to the contribution of the Unique Therapeutic Project, the accomplishment of educational activities and the development of practices with an appreciation of intersectoriality and integrality. Conclusion: It is considered that the experience analyzed projected important advances, encompasses actions of health education, intersectoriality and reorganization of actions of professional practices. However, it has not yet been able to modify the work processes, which would require the execution of a project articulated between different professionals and the cooperation of the hospital management staff.
\end{abstract}

\section{DESCRIPTORS}

Human Resources.Professional Training.Continuing Education.

\footnotetext{
Pesquisadora do Setor de Estudos e Pesquisas em Saúde e Serviço Social. Universidade Federal da Paraíba - UFPB. João Pessoa. Paraíba. Brasil.

Professora do Programa de Pós-graduação em Serviço Social. Universidade Federal da Paraíba - UFPB. João Pessoa. Paraíba. Brasil.

Doutorando do Programa de Pós-graduação em Enfermagem. Universidade Federal da Paraíba - UFPB. João Pessoa. Paraíba. Brasil.

Professora Titular do Departamento de Serviço Social. Universidade Federal da Paraíba - UFPB. João Pessoa. Paraíba. Brasil.

5 Doutoranda do Programa de Pós-graduação em Ciências da Religião, Universidade federal da Paraíba-UFPB. João Pessoa. Paraíba. Brasil.
} 
$\mathrm{N}$ o Brasil, o atendimento na área da saúde é reconhecidamente controverso, seja no que diz respeito à relação desigual entre demanda e oferta dos serviços, seja em relação às condições precárias dos equipamentos que prestam tal atendimento. Outro aspecto problemático diz respeito às deficiências detectadas na formação profissional. Estudos em torno da educação permanente para o SUS vêm constatando que o perfil de formação dos profissionais da área de saúde não é adequado para prepará-los para uma atuação na perspectiva da integralidade e de práticas que envolvam ações de promoção, proteção, prevenção, cura e reabilitação ${ }^{1}$.

Para superação desta realidade faz-se necessária a elaboração e a adoção de novas referências e estratégias para a gestão e para a organização do trabalho em saúde que contribuam para substituição da categorização, da simplificação dos saberes e da separação entre sujeito e objeto, buscando a configuração de perspectivas que considerem a complexidade dos processos de saúde-doença ${ }^{2}$.

Neste sentido, a Residência Multiprofissional em Saúde (RMS) foi instituída no cenário brasileiro como uma estratégia do Ministério da Saúde no intuito de possibilitar mudanças no modelo técnico-assistencial e na atuação multiprofissional inserida em um processo de Educação Permanente em Saúde.

O estímulo ao projeto da Residência Multiprofissional em Saúde está associado a um conjunto de ações que visam provocar mudanças nos cursos de graduação em saúde, de modo a engendrar mudanças que corroborem para uma abordagem integral do processo saúde-doença, a melhoria dos serviços prestados à população e, assim, fortalecer e qualificar o SUS, seus princípios e diretrizes ${ }^{3}$

As Residências Multiprofissionais foram criadas a partir da promulgação da Lei $n^{\circ} 11.129$ de 30 de junho 2005 com a criação da Comissão Nacional de Residência Multiprofissional (CNRMS). Trata-se de uma modalidade de Pós-Graduação lato sensu que possui como objetivos: acrescentar ao serviço de saúde a formação e desenvolvimento dos trabalhadores; articular o trabalho em equipe; ampliar o conjunto de ações e serviços da atenção em saúde; humanizar a assistência; e promover a integralidade ${ }^{3,4}$.

Neste sentido, para regulamentação da Residência Multiprofissional em Saúde foram elaboradas pelo Ministério da Saúde as portarias $n^{\circ} 1.111$, de 5 de julho de 2005 , e no 1.143 , de 7 de julho de 2005, além da Portaria Interministerial $\mathrm{n}^{\circ}$ 2.117, de 3 de novembro de 2005.

O Programa de Residência Multiprofissional configura-se como objeto deste estudo, que por sua vez teve por finalidade investigar o desenvolvimento do processo de implementação do Programa, a partir da análise de uma experiência prática em um hospital universitário. Para tanto, optou-se por realizar uma pesquisa exploratória, conjuminandose os levantamentos bibliográficos e documentais à realização de entrevistas por meio das quais se pudesse coletar a concepção dos participantes preceptores e tutores - acerca das fragilidades e potencialidades que permearam tal processo, de modo a caracterizá-lo.

De acordo com o artigo $11^{\circ}$ da Resolução da Comissão Nacional de Residência Multiprofissional em Saúde - CNRMS $n^{\circ} 2$ de 13.04.2012, a função de tutor é caracterizada por atividade de orientação acadêmica de preceptores e residentes, estruturada preferencialmente nas modalidades de tutoria de núcleo e tutoria de campo, exercida por profissional com formação mínima de mestre e experiência profissional de, no mínimo, 03 (três) anos. Desta forma, cabe ao tutor a função de integrar os conceitos advindos do ensino e da prática profissional. Além disso, o tutor tem a função de mediar e garantir a integração entre as unidades acadêmicas e os serviços envolvidos nos programas de residência.

No artigo $13^{\circ}$ da mesma Resolução, a função de preceptor é sumariada pela supervisão direta das atividades práticas realizadas pelos residentes nos serviços de saúde onde se desenvolve o Programa, exercida por profissional vinculado à instituição formadora ou executora, com formação mínima de especialista devendo o preceptor, conforme indicado no parágrafo $1^{\circ}$, ser necessariamente da mesma área profissional do residente sob sua supervisão, estando presente no cenário de prática.

\section{MATERIALE MÉTODOS}

O presente artigo deriva de uma pesquisa de cunho quantitativo e qualitativo, realizada nos meses de janeiro a março de 2012 junto aos preceptores e tutores do Programa de Residência Integrada Multiprofissional em Saúde Hospitalar (Rimush) desenvolvida no Hospital Universitário Lauro Wanderley (HULW), localizado em João Pessoa, Paraíba.

O percurso metodológico escolhido para 
alcançar o trabalho proposto encontra-se estruturado numa pesquisa exploratória. Foram utilizados como instrumentos de coleta de dados secundários: os levantamentos bibliográfico e documental, incluindo o documento oficial do Programa. Estes orientaram a elaboração do questionário estruturado que se configurou como instrumento para obtenção dos dados primários, bem como a definição das principais categorias a serem trabalhadas, conformando-se como descritores: Recursos Humanos, Capacitação Profissional, Educação Continuada.

Os dados foram analisados numa abordagem quali-quantitativa, atingindo-se o aspecto quantitativo a partir do estudo do fragmento percentual das variáveis do universo de profissionais envolvidos na Rimush. Para interpretação qualitativa dos dados primários, os depoimentos dos entrevistados foram analisados a partir da literatura empreendida para consecução da pesquisa. Para garantia do sigilo absoluto, os nomes dos entrevistados foram omitidos, sendo registrados por algarismos arábicos.

Sobre o Processo de Criação e Implementação da Residência Multiprofissional no Hospital Universitário Lauro Wanderley

Na Paraíba, o Hospital Universitário Lauro Wanderley (HULW), pertencente à Universidade Federal da Paraíba (UFPB) e referência na assistência à saúde estadual, foi o pioneiro a oferecer a modalidade de Residência Multiprofissional.

As atividades da Residência Integrada Multiprofissional em Saúde Hospitalar (Rimush) iniciaram-se em abril de 2010, tendo como objetivo geral: especializar profissionais das diferentes áreas que se relacionam com a saúde, através da formação em serviço, com a finalidade de atuar em equipe, de forma multiprofissional e interdisciplinar, articulando os diversos níveis de atenção, equipamentos e a gestão do Sistema Único de Saúde, além de fornecer subsídios para o desenvolvimento de pesquisas, aprimorando e qualificando a capacidade de análise, de enfrentamento e proposição de ações que visem concretizar os princípios e diretrizes do SUS, com especial destaque para a promoção da atenção integral ${ }^{5}$.

A equipe matricial responsável pela elaboração e implementação do projeto da Residência buscou apresentá-lo e construí-lo de modo inovador, no intuito de atender às exigências e necessidades de saúde da população no que se refere à formação de profissionais críticos e reflexivos, com aptidão para lidar com questões específicas inerentes à atenção à saúde do indivíduo, família e comunidade, dentro de uma abordagem multidisciplinar e interdisciplinar, como preconiza o SUS.

A referida equipe foi constituída por 90 (noventa) profissionais, deste quantitativo apenas 21 (vinte e um) deles continuaram no Programa até o período de sua implementação, sendo: 18 (dezoito) preceptores e 3 (três) tutores. Um ano após o início de suas atividades, o Programa sofreu a desistência de outros 5 (cinco) preceptores, cujo universo - até o momento da pesquisa - era constituído por 13 (treze) preceptores e 3 (três) tutores, oriundos das profissões contempladas pela Residência, a saber: assistentes sociais, enfermeiros, farmacêuticos, fisioterapeutas e nutricionistas.

A Rimush foi subdividida em três ênfases de saber e práticas: "Atenção ao Paciente Crítico", "Atenção à Saúde Cardiovascular do Adulto" e "Atenção à Saúde do Idoso". As atividades práticas e teóricas foram realizadas de acordo com as propostas de especialização específicas por ênfase, entretanto cabe ressaltar que a ênfase à Saúde Cardiovascular do Adulto contou com algumas dificuldades devido ao declínio da cirurgia cardíaca no estado, em 2010, e à desativação temporária do serviço no HULW.

Os módulos teóricos foram desenvolvidos sob a forma de reuniões de preceptoria, seminários integradores e sessões tutoriais, bem como acompanhados pela participação de profissionais que integravam serviços de saúde da atenção média e básica. As reuniões de preceptoria foram realizadas por ênfase e consistiam em estratégias que rotineiramente subsidiavam as discussões do grupo, nestes momentos eram trazidas discussões referentes às experiências vivenciadas nos campos de práticas, eram realizadas apresentações de artigos científicos, além disso, eram dados os encaminhamentos necessários ao desenvolvimento das atividades na Residência.

Os seminários integradores objetivaram a articulação das experiências dos participantes por meio da problematização das atividades práticas dos residentes nos serviços de saúde, esses momentos contavam com a participação das três ênfases.

As sessões tutoriais contavam com 
discussões de casos hipotéticos e/ou casos experenciados pelos residentes, no intuito de instigar debates em torno do reconhecimento do problema e na elaboração de propostas de solução e intervenção.

\section{Apresentando os Protagonistas da Pesquisa}

O universo do estudo aqui retratado foi constituído por todos os preceptores e tutores que fizeram parte do Programa de Residência por no mínimo um ano, totalizando 20 (vinte) profissionais. A amostra constituiu-se de 18 (dezoito) profissionais que concordaram em participar da pesquisa e estiveram presentes na instituição no período da coleta dos dados, configurando uma amostra de $98 \%$.

Deste quantitativo $77 \%$ foram do sexo feminino e $22 \%$ do sexo masculino. A faixa etária variou de 29 a 56 anos, sendo que a média prevalente foi de 42 (quarenta e dois) anos. 55\% da amostra apresentou o tempo de formação acadêmica superior a 20 (vinte) anos, tendo $27,5 \%$ apresentado entre 10 a 20 anos e $16 \%$ entre 5 a 10 anos. $89 \%$ da amostra apresentou o título de especialista enquanto $44,6 \%$ o título de mestre. A função de preceptor foi apresentada por $89 \%$ da amostra e a de tutor por $11 \%$.

\section{RESULTADOS}

Ao longo da pesquisa foi possível observar que o processo de implementação da Residência Multiprofissional foi permeado por algumas dificuldades, dentre elas foram mencionadas: a estrutura organizacional do Programa; o número de profissionais responsáveis por garantir o acompanhamento dos residentes; a falta de remuneração para preceptores/ tutores; e a ausência de normas e rotinas claras e eficientes, bem como de diretrizes e metas para os serviços e seus respectivos profissionais; a crise de financiamento enfrentada pelos HUs; o envelhecimento dos profissionais da instituição; e o fato de não haver integração entre a Residência Multiprofissional e a Residência Médica.

No que concerne às potencialidades, as mesmas foram relacionadas aos objetivos preconizados pelo Programa, os quais representariam novas possibilidades para atuação no SUS, referendados pela valorização dos diferentes sujeitos implicados nos processos de trabalho no âmbito da saúde. Nota-se que houve o entendimento de que essas propostas implicariam em melhor atendimento dos usuários, além de facilitarem a integralidade, contribuindo deste modo para o fortalecimento do SUS.

Houve também a referência de mudança de paradigma através da capacitação de profissionais, no intuito de possibilitar o desenvolvimento de práticas diferenciadas no trabalho na saúde, já que a Residência proporcionaria o espaço para discussões com base na multiprofissionalidade.

Os depoimentos que seguem reforçam tais informações:

"Há uma interação entre os profissionais, fazendo com que estes tenham a real noção do problema de cada paciente" (Entrevistado 11).

"Mudança de paradigma. Formação de profissionais que trabalhem com enfoque multidisciplinar, passando a servir de exemplo para os profissionais que trabalham em Hospitais Universitários" (Entrevistado 16).

"Desenvolver nos profissionais a concepção multiprofissional; fortalecimento do SUS; integralidade da assistência; a forma de construção dos pilares da Residência"(Entrevistado 17).

Entretanto, ao serem questionados se a Rimush recebeu o apoio institucional por parte das Diretorias e do corpo administrativo em geral, aqui destacado como necessário para o desenvolvimento de suas propostas, observou-se uma baixa interação entre as propostas do Programa e a realidade institucional cotidiana. Desta forma, $72,30 \%$ apontaram não haver reciprocidade e relacionamento entre os membros da administração e a equipe da Rimush, enquanto $11,10 \%$ afirmaram sua existência, $11,10 \%$ que ela se mostra apenas parcialmente e 5,50\% que não souberam responder.

Os depoimentos que seguem complementam estes dados:

"Parcialmente. Acredito que se a instituição tivesse designado profissionais exclusivos para a residência, inclusive professores, o desenvolvimento seria outro" (Entrevistado 4).

"Não. Não houve inicialmente liberação de carga horária para a exclusiva destinação 
às ações da Rimush, as salas da Rimush não tinham os recursos necessários (computadores, estabilizadores, ar condicionado, livros)"(Entrevistado 13).

"Sim. Entendo que tivemos a oportunidade de adentrar o sistema organizacional nos segmentos: educacional; assistencial e administrativo" (Entrevistado 14).

No que tange ao apoio das Coordenações por núcleo profissional, constatou-se que $55,50 \%$ expuseram ter havido apoio por parte de suas Coordenações para o desenvolvimento de atividades de preceptoria e de tutoria na Rimush, porém 39\% não receberam este suporte e $5,50 \%$ não souberam ou não quiseram responder.

Por sua vez, $11,10 \%$ não visualizaram barreiras para o desenvolvimento do trabalho multiprofissional, enquanto $77,80 \%$ as identificaram, o que implicaria em dificuldades para a operacionalização das propostas da Rimush.

Em menção à crença na minimização de barreiras ao trabalho multiprofissional decorrentes da implantação da Rimush, $81,20 \%$ dos discursos apontaram para a percepção de grande contribuição para a consecução do trabalho na perspectiva da multiprofissionalidade.

Faz-se mister destacar que não houve a justificativa por parte dos $12,50 \%$ da amostra que consideraram que a Residência não contribuiu para a minimização das barreiras detectadas.

Concomitante à crença na minimização de impedimentos, $83,40 \%$ asseveraram perceber inovação no trabalho como resultante da implementação da Rimush no HULW. Em contrário, apenas $5,50 \%$ dos entrevistados, restando ainda $11,10 \%$ que não souberam ou não quiseram responder.

As falas abaixo elucidam tais percentuais:

"Sim. Entrosamento das equipes, introdução da intersetorialidade atenta aos usuários na sua integralidade" (Entrevistado 1).

"Sim. A Residência em si mesmo já o é. Vivências na construção do Projeto Terapêutico Singular" (Entrevistado 3).

"Sim. Mesmo ainda com muita coisa para conquistar vejo que a instituição começa a perceber e sentir a necessidade da multiprofissionalidade" (Entrevistado 10).
"Não. Pelo pouco conhecimento que possuo com relação a Residências, não há nada de inovador" (Entrevistado 15).

Observou-se a expressiva concordância de que houve algo de inovador no trabalho empreendido pelas equipes partícipes da Residência Multiprofissional, entendendo-se que esta representaria inovação ao oferecer a possibilidade de um processo formativo favorecedor e incentivador do trabalho na perspectiva da multiprofissionalidade e da interprofissionalidade, o que redundaria na valorização dos diversos sujeitos implicados no cuidado em saúde, utilizando-se, para tanto, de estratégias idealizadas, elaboradas e preconizadas pelo SUS.

Como exemplo disto houve a realização do Projeto Terapêutico Singular (PTS), preconizado pelo Ministério da Saúde. Este projeto foi aplicado junto às equipes da Residência tendo primordialmente contribuído para a integração da equipe, por favorecer o entendimento da importância de se trabalhar interprofissionalmente; facilitar a compreensão da importância das distintas categorias profissionais envolvidas no processo de disseminação do cuidado em saúde, além de implicar na potencialização da capacidade para delimitar espaços e traçar possibilidades no que concerne à elaboração das condutas terapêuticas.

Por favorecer a comunicação, o PTS também colaborou para que a equipe ultrapassasse os espaços da Residência, permitindo-se conhecer e ser conhecida dentro da dinâmica institucional através da interação com os demais profissionais.

Como outras importantes inovações no contexto institucional, foram citadas: a introdução da intersetorialidade; o oferecimento de treinamento a pacientes/profissionais; o planejamento da assistência; além do incentivo às ações de educação em saúde.

\section{DISCUSSÃO}

Como uma das principais constatações, concluiu-se que a estrutura organizacional da Residência representou uma grande fragilidade, ainda que em muitos depoimentos isto tenha sido relacionado ao fato de tratar-se de um projeto pioneiro, ao que se somou o desconhecimento de suas propostas e a falta de referências que pudessem contribuir para a facilitação de seu desenvolvimento institucional. 
O número de profissionais envolvidos também se configurou em um sério problema, pois implicou em grande dificuldade para garantir o alcance das propostas do Programa, dado o déficit de preceptores, tutores e principalmente do corpo docente.

A falta de remuneração para preceptores e tutores, que também se constituiu como importante dificuldade, pode ser considerada como um dos fatores responsáveis pela significativa evasão destes profissionais que, juntamente com a não liberação inicial da carga horária para o desempenho das funções na Rimush decorreram em evasão de parte daqueles que organizaram a proposta. Já a ausência de normas e rotinas claras, mencionada como uma das fragilidades do Programa, tanto pode indicar falhas no planejamento inicial, quanto pode ser também associada ao seu pioneirismo, sendo, deste modo, factível de superação.

O período de realização da pesquisa foi marcado pela crise de financiamento que envolveu os hospitais universitários no cenário brasileiro, a qual pôde ser constatada pelo alto índice de profissionais terceirizados, pela escassez de recursos humanos e materiais, além dos problemas infraestruturais observados nos cotidianos de trabalho. Tais problemas culminaram no processo de terceirização que os hospitais universitários experimentaram nos últimos anos por intermédio da adesão à Empresa Brasileira de Serviços Hospitalares, criada por intermédio da medida provisória $n^{\circ} 520$ e da Lei 12.550 , de 15 de dezembro de 2011.

Observa-se que o processo de expansão dos Programas de Residências Multiprofissionais ocorre em um contexto de contrarreformas dos SUS e de fragilização da seguridade social brasileira. Esse cenário pode ser caracterizado pelo sucateamento dos serviços, pela insuficiência de profissionais e pela precarização das condições e relações de trabalho, podendo acarretar no âmbito dessa modalidade formativa, ações voltadas para o produtivismo, contribuindo para secundarizar 0 ensino e a pesquisa ${ }^{6}$.

Assim, compreende-se que é preciso observar os espaços onde as residências estão inseridas, bem como o modelo de gestão assumido pelas coordenações dos programas, para que a qualificação profissional desejada seja assegurada de modo ético, qualificado e responsável, já que o produto final, isto é, o perfil dos profissionais, reflete uma estreita articulação com o modo como o processo pedagógico foi conduzido 1 .

Quanto ao envelhecimento dos profissionais da instituição, entende-se que este pode ser atribuído à ausência de concursos com número adequado de vagas, isto somado ao dimensionamento insatisfatório de pessoal. Há que se destacar que o retardo no investimento em contratação de novos profissionais e mesmo na qualificação dos já existentes pode implicar em práticas obsoletas, dificuldade em adequar-se a um novo contexto de relações sociais, corroborando para a manutenção de um conservadorismo persistente.

Outra dificuldade apontada foi a não integração entre a Residência Multiprofissional e a Residência Médica. Quanto a isto, percebe-se a importância de que haja a elaboração de estratégias que objetivem tal integração, pois já que há o debate em torno de mudanças nas práticas em saúde, e há a elaboração de estratégias para a superação do atual modelo hegemônico, entende-se que estas devem alcançar todos os profissionais envolvidos nos processos de trabalho em saúde. Assevera-se diante disto a importância da gestão para empreender e/ou incentivar ações que visem tal articulação.

Em menção às potencialidades, foi possível perceber a credibilidade que estes profissionais deram aos objetivos do Programa, isto atestou o reconhecimento da necessidade de redirecionar uma prática profissional mais adequada às necessidades dos usuários, além de confluir para a defesa dos princípios do SUS. Também foi possível perceber o anseio por mudanças por intermédio da multiprofissionalidade.

No tocante ao apoio institucional, registrouse a incipiência de tal apoio direcionado à Residência, o que contribuiu para agravar a dificuldade no desenvolvimento de suas propostas, bem como sua reprodução e consolidação, posto que sem a participação da gestão no processo, pensar em mudanças torna as propostas e ações a elas tendentes obtusas e conflituosas.

Por se tratar de uma proposta inovadora com o objetivo de empreender práticas pouco visualizadas no âmbito institucional, é comum que 
os integrantes da Residência lidem diariamente com a geração de conflitos e tensões. Neste sentido, considera-se a importância da constituição e manutenção de espaços estimulados pelos gestores com o objetivo de assegurar a abordagem dos problemas. Acredita-se que tentativas de mudanças sem a alteração da cultura organizacional, invariavelmente tropeçam, demandam muito mais tempo ou falham. Por outro lado, quando bem conduzidas, proporcionam o contexto adequado para a condução da estratégia da organização, além de implicar em menor carga psíquica e consequentemente menor sofrimento dos trabalhadores ${ }^{7}$.

Cabe salientar que o pífio apoio direcionado à Rimush também se contrapõe ao que é definido pelo Decreto $n^{\circ} 7.082^{8}$ de 27 de janeiro de 2010 que define como sendo um dos objetivos dos hospitais universitários: implantar e implementar a Residência Multiprofissional, estimulando o trabalho em equipe multiprofissional e contribuindo com a qualificação de recursos humanos especializados.

Por outro lado, houve o registro de que $55,50 \%$ dos profissionais envolvidos obtiveram o apoio de suas Coordenações, o que se configurou como um fator positivo, e mesmo nos casos em que não houve o envolvimento por parte das chefias, foi observado o entendimento da importância da Residência. Por sua vez, a frequência de $39 \%$ de profissionais que não obtiveram o apoio e nem o reconhecimento da importância da Rimush expressou que há ainda muito espaço para ser conquistado e revelou a existência de uma cultura organizacional pouco afeita a mudanças, com perfil centralizador e autoritário no que tange aos processos decisórios ${ }^{9}$.

Quanto à detecção de barreiras para realização do trabalho na perspectiva da multiprofissionalidade, sabe-se que tal trabalho envolve mudanças nas relações por intermédio das quais se desempenham os processos de trabalho em saúde, isto aliado à rígida delimitação nos campos de saberes e práticas de cada categoria profissional. Deste modo pressupõe-se que a realização do trabalho multiprofissional não seja um processo pacífico.

Entende-se por atuação multiprofissional uma modalidade de trabalho coletivo que se baseia na comunicação e no diálogo como instrumentos fundamentais para a interação entre os agentes e a troca de saberes técnicos, visando à interação entre os diversos profissionais em prol de um objeto comum, no caso, o atendimento das demandas e necessidades dos usuários ${ }^{10}$.

Articular estes distintos aspectos não é uma tarefa simples, pois requer contínuo esforço e dedicação. Apesar de inquestionável relevância para a melhoria institucional, as mudanças causam os mais diferentes tipos de reação dos trabalhadores. Enquanto alguns trabalhadores podem enxergar nas mudanças uma oportunidade de desempenharem práticas mais condizentes com as necessidades dos usuários, outros podem enxergar como "um trabalho a mais", pelo fato de terem que sair de sua "zona de conforto".

Assim, é preciso conceber o mundo do trabalho como espaço de aprendizagem constante, o que implica reconhecer e problematizar a existência de espaços que são favorecedores de recriações sociais, ainda que seja necessário admitir também que existem serviços de saúde que são espaços sociais mortíferos, ou seja, que impedem e neutralizam qualquer movimento de criação ${ }^{11}$.

Por outro lado, o registro de $11,10 \%$ dos profissionais afirmando não visualizar tais barreiras informa a existência de setores na instituição que desenvolvem uma prática na perspectiva da multiprofissionalidade, configurando uma contracultura organizacional. Neste contexto, a Residência deve contribuir no intuito de fortalecer estas ações, além de incitar a construção e o desenvolvimento de um projeto mais amplo, com práticas que possam dirimir as barreiras detectadas e citadas anteriormente.

Neste sentido, houve o registro de $81,20 \%$ da amostra indicando a contribuição da Residência Multiprofissional para minimizar as dificuldades enfrentadas, o que representa um dado de considerável relevância, já que as Residências pretendem produzir mudanças estruturais nas formas de se operar o trabalho em saúde, mudanças estas há muito desejadas e defendidas pelos segmentos que lutaram e lutam pela transformação do modelo assistencial aos moldes previstos pela Reforma Sanitária e pelo SUS. Para isto, elas empreendem uma proposta de formação que pretende produzir abalos nos territórios vigentes e 
abertura para novas composições que problematizem os saberes e as práticas ${ }^{12}$.

Corroborando com tais afirmações, observou-se a expressiva concordância, por parte de $83,40 \%$ da amostra, de que houve a percepção de inovação no trabalho empreendido por intermédio da Rimush.

A elaboração do PTS foi considerada como uma destas inovações. O PTS consiste em um conjunto de propostas de condutas terapêuticas articuladas para um sujeito individual ou coletivo, resultado da discussão coletiva e geralmente dedicado a situações mais complexas. Conta com quatro etapas, a saber: diagnóstico: fase em que é realizada a avaliação a respeito das demandas do usuário; definição de metas: etapa em que são realizadas as propostas de curto, médio e longo prazo a serem apresentadas ao usuário; divisão de responsabilidades: definição dos responsáveis por realizar as propostas; reavaliação: momento em que se discute a evolução e se serão feitas alterações nas propostas elaboradas ${ }^{13}$.

Como outra importante inovação foi mencionada a intersetorialidade, que possui como preceito a reestruturação e a reunião de vários saberes e setores no sentido de um olhar mais adequado e menos falho a respeito de um determinado objeto, proporcionando uma melhor resposta aos possíveis problemas encontrados no dia-a-dia, o que foi viabilizado no Hospital por meio da Residência que funcionou como uma ponte para articulação do Hospital com outros níveis de atenção à saúde, a partir da realização de rodízios na atenção básica e secundária ${ }^{14}$.

Entretanto, foi possível observar que a compreensão da intersetorialidade não ultrapassou o movimento endógeno do setor saúde. Isso representou uma falha, haja vista a importância da compreensão de saúde enquanto conceito ampliado, o que pressupõe a necessidade de articulação dos distintos setores da sociedade para empenho da produção da saúde. Em contrapartida, em termos da política setorial da saúde, também sinalizou um avanço, tendo em vista a dificuldade que representa a articulação entre os distintos níveis de atenção.

Por fim, e não menos importante, foi citado também como significativo e inovador o incentivo às ações de educação em saúde, sejam as destinadas aos profissionais, sejam as voltadas para os usuários.

Por ações de educação em saúde entendese como sendo um processo de capacitação de indivíduos e grupos para assumir a solução dos problemas de saúde, este processo inclui o crescimento dos profissionais de saúde por intermédio de reflexão conjunta sobre o trabalho que desenvolvem e suas relações com a melhoria das condições de saúde da população. Ressaltase que estas ações devem ocorrer em todo e qualquer contato entre o profissional de saúde e a população ${ }^{15}$.

É importante ressaltar a importância do processo de educação para o cuidado em saúde, ainda que não se possa descurar das dificuldades para garantia de sua realização. Entende-se que o dado registrado representa um avanço, pois se observa o desafio que representa a realização dessa atividade, que tem sido secundarizada devido haver uma valorização no tocante à realização de atendimentos individuais focados aos aspectos assistenciais $^{16}$.

Esse não é o único desafio que envolve o processo formativo por intermédio da Residência Multiprofissional. A materialização da integralidade, intersetorialidade e interdisciplinaridade, ainda que seja um dos objetivos do programa, tem constituído como um importante desafio. Além disso, a carga horária de 60 horas tem sido apontada como uma problemática. Por se tratar de uma carga horária exaustiva, acredita-se que ela pode comprometer a qualidade da formação, pois dificulta a reflexão dos residentes em relação às ações desenvolvidas, o que pode contribuir para a formação de um profissional imediatista e tecnicista. Para a superação de tais desafios é imprescindível a articulação e organização política ${ }^{16}$.

É nesse contexto que a Rimush tem desenvolvido suas atividades. Atualmente o programa encontra-se na formação da sexta turma e contou com algumas mudanças. Houve ampliação das vagas ofertadas e foram incluídas as seguintes profissões: odontologia, fonoaudiologia, terapia ocupacional e psicologia. Além disso, a ênfase Atenção à Saúde Cardiovascular foi substituída pela ênfase Atenção à Saúde da Criança e do Adolescente. Em relação às dificuldades apresentadas no estudo, observa-se no contexto 
atual que algumas foram superadas, como aspectos relacionados à estrutura organizacional, a oferta de módulos teóricos e maior engajamento de preceptores. Todavia, outros persistem.

\section{CONCLUSÃO}

De acordo com os dados obtidos na pesquisa, foi possível perceber que a Residência Multiprofissional enfrentou obstáculos principalmente de ordem estrutural e organizacional, os quais complicaram sobremaneira a plena efetivação de suas propostas. Porém, tendo em vista o seu pioneirismo, acredita-se que, apesar de todas as dificuldades detectadas, a Residência Multiprofissional no HULW conseguiu imprimir o engendramento de uma nova concepção de trabalho em saúde. Entretanto, as ações desenvolvidas neste período, em sua maioria, não são sustentáveis sem que haja novas ações para ressignificá-las, reafirmá-las. Quanto a isso, afirma-se a importância das turmas subsequentes.

Ademais, é importante ter ciência de que a Residência Multiprofissional foi idealizada e desenvolvida para atuar a partir de fragilidades existentes nos processos de trabalho em saúde, deste modo não há como concebê-la imune a obstáculos. Todavia, acredita-se que a realização de oficinas avaliativas, no intuito de identificar falhas e apontar possíveis soluções, poderia amenizar tais obstáculos e consequentemente potencializar suas propostas.

Considera-se que a experiência analisada projetou avanços importantes, pois engendrou ações de educação em saúde, intersetorialidade e reorganização de ações nas práticas profissionais. Contudo, ainda não conseguiu modificar os processos de trabalho, tangenciados por uma ótica verticalizada, burocratizada e com foco na figura do médico, cuja ruptura exige um projeto articulado entre as distintas profissões e com cooperação da gestão hospitalar, de ordem permanente e continuada para que os avanços projetados possam, de fato, ser implementados e consolidados, constituindo uma nova cultura organizacional.

\section{REFERÊNCIAS}

1. Cavalcanti PB, Lucena CM, Lucena PLC, Miranda APRS. Formação de recursos humanos para o sus: uma análise da residência multiprofissional. Interface. 2015; 12(2): 1-22.

2. Morin E. A cabeça bem-feita: repensar e reformar o pensamento. Rio de Janeiro: Bertrand Brasil; 2000.

3. Ministério da Saúde. Residência multiprofissional em saúde: experiências, avanços e desafios. Brasília: Ministério da Saúde, Secretaria de Gestão do Trabalho e da Educação na Saúde; 2006.

4. Ministério da Educação. Residência multiprofissional. Brasília: MEC; 2012.

5. Hospital Universitário Lauro Wanderley. Residência Integrada Multiprofissional em Saúde Hospitalar RIMUSH. Processo seletivo público edital 01/2010. João Pessoa; 2010.

6. Castro MM. O serviço social nos programas de residência em saúde: resultados iniciais do mapeamento da ABEPSS. Rev Temporális. 2013; 13(26): 153-171.

7. Mamede AAC. A influência da cultura organizacional nos processos de mudança. Sebrae Biblioteca on line 2009.

8. Brasil. Portaria Interministerial n. 1006/MEC/MS, de 27 de maio de 2004. Programa de Reestruturação dos Hospitais Universitários de Ensino do Brasil, visando reorientar e, ou formular a política nacional para o setor. Diário Oficial da União 2004; 27 maio.

9. Miranda APRS. Inserção do serviço social nos processos de trabalho em serviços públicos de saúde - concepções e práticas: estudo de caso em um hospital universitário - João Pessoa-PB [tese]. São Paulo: Programa de Estudos Pós-Graduados em Serviço Social, Pontifícia Universidade Católica de São Paulo; 2011. $p(300 p)$

10. Peduzzi M. Equipe multiprofissional de saúde: a interface entre trabalho e interação. [tese]. Campinas: Programa de Pós-Graduação em Saúde Coletiva do Departamento de Medicina Preventiva e Social, Universidade Estadual de Campinas; 1998. p(230p)

11. Silva QTA, Cabalero RMS. A micropolítica da formação profissional na produção do cuidado: devir-residência. In: Fajardo AP, Rocha CMF, Pasine VL. Residências em Saúde: Fazeres e Saberes na Formação em Saúde. Porto Alegre: Hospital Nossa Senhora da Conceição; 2010: 61-74.

12. Pasine VL, Guareschi NMF. Problematizando a produção de saberes para a invenção de fazeres em saúde. In: Fajardo AP, Rocha CMF, Pasine VL. Residências em Saúde: Fazeres e Saberes na Formação em Saúde. Porto Alegre: Hospital Nossa Senhora da Conceição; 2010: 145-160.

13. Ministério da Saúde. Clínica ampliada, equipe de referência e projeto terapêutico singular. Brasília: Secretaria de Atenção à Saúde/Núcleo Técnico da Política Nacional de Humanização; 2007. 
14. Macedo PCM. Desafios atuais no trabalho multiprofissional em saúde. Rev SBPH 2007; 10(2): 3341. Mamede AAC. A influência da cultura organizacional nos processos de mudança. Sebrae Biblioteca on line 2009.

15. Ministério da Saúde. Educação em Saúde: planejando as ações educativas. Teoria e práticas. Manual para operacionalização das ações educativas no SUS. Brasília: Secretaria de Atenção Básica; 1997.

16. Guerra TMSG, Maria DHC. Formação profissional da equipe multiprofissional em saúde: a compreensão da intersetorialidade no contexto do SUS. Rev Texts \& Contexts. 2017; 16(2): 454-469.

\section{CORRESPONDÊNCIA}

Carla Mousinho Ferreira Lucena

Rua Professor Manoel Viana, 54, Castelo Branco CEP: $58050-280$

João Pessoa - Paraíba - Brasil

E-mail: carlamousi@hotmail.com 